CrossMark \& click for updates

Cite this: J. Mater. Chem. C, 2016, 4, 2731

Received 23rd November 2015, Accepted 10th February 2016

DOI: $10.1039 / c 5 t c 03933 a$

www.rsc.org/MaterialsC

\title{
Recent advances in twisted intramolecular charge transfer (TICT) fluorescence and related phenomena in materials chemistry
}

\author{
Shunsuke Sasaki, ${ }^{a}$ Gregor P. C. Drummen ${ }^{\star b}$ and Gen-ichi Konishic
}

\begin{abstract}
Twisted intramolecular charge transfer (TICT) is an electron transfer process that occurs upon photoexcitation in molecules that usually consist of a donor and acceptor part linked by a single bond. Following intramolecular twisting, the TICT state returns to the ground state either through red-shifted emission or by nonradiative relaxation. The emission properties are potentially environment-dependent, which makes TICT-based fluorophores ideal sensors for solvents, (micro)viscosity, and chemical species. Recently, several TICT-based materials have been discovered to become fluorescent upon aggregation. Furthermore, various recent studies in organic optoelectronics, non-linear optics and solar energy conversions utilised the concept of TICT to modulate the electronic-state mixing and coupling on charge transfer states. This review presents a compact overview of the latest developments in TICT research, from a materials chemistry point of view.
\end{abstract}

\footnotetext{
${ }^{a}$ Department of Organic and Polymeric Materials, Tokyo Institute of Technology, Okayama, Tokyo, Japan

${ }^{b}$ Bionanoscience and Bio-Imaging Program, Cellular Stress and Ageing Program, Bio\&Nano-Solutions, Bielefeld, Germany.E-mail: gpcdrummen@bionano-solutions.de ${ }^{c}$ PRESTO (Japan) Science and Technology Agency (JST), Kawaguchi, Saitama, Japan
}

\section{Introduction}

Photophysical properties of some fluorescent molecules are well-known to be sensitive to local steric environments. Fluorescence phenomena that involve energy transfer, partial

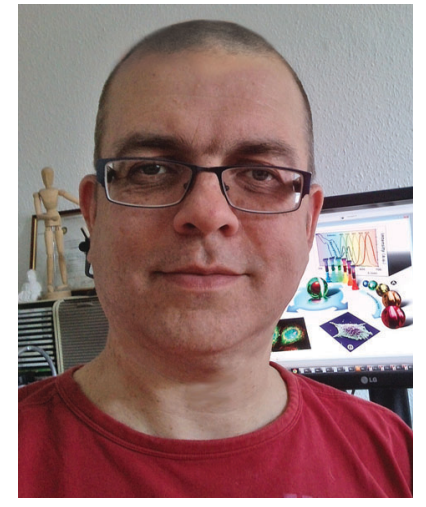

Gregor Drummen
Gregor Drummen was born in Maastricht, the Netherlands, in 1968. He received bachelor degrees in Chemical Engineering and Bioprocesstechnology from Zuyd University (NL), post-graduate diplomas in Biotechnology and Biomedical Science from Teesside and Ulster Universities (UK), a MSc in Chemistry, specializing in Cell and Chemical Biology, and a PhD degree in Biochemistry (Professors Henk van den Bosch and Jos Op den Kamp) from the Bijvoet Center for Biomolecular Research (formerly CBLE) and the Institute for Biomembranes of Utrecht University (NL). After post-doctoral and senior scientist appointments with various Multinational and Biotech companies and the University of Duisburg-Essen (Germany), he currently runs Bio\&Nano-Solutions and the $L A B^{3} B I O$ project. His research interests include oxidative stress and antioxidants, extracellular vesicles, renal disease, advanced optical imaging techniques and fluorescent probes, and (bio)nanotechnology, including Laser ablation synthesis in solution (LASiS) and non-linear optics. 
charge or pure electron transfer, intermolecular proton transfer, excimer/exciplex formation and $\mathrm{J}$ - or $\mathrm{H}$ - aggregates are sometimes affected largely by, for instance, relative distance/orientation of fluorophores, conformation of individual fluorophores and their mobility. These fluorophores are not only versatile reporters for steric environments at mesoscopic to microscopic scales, but also expected to be a motif of novel functional molecules because certain steric restrictions or well-ordered arrangement of fluorophores may activate unprecedented photophysical properties.

We start from energy transfer phenomena such as Förster Resonance Energy Transfer (FRET) because these phenomena are photophysical processes generally known to researchers and are often employed in applications relevant to material and life sciences. ${ }^{1}$ Though energy transfer processes are different from charge transfer process in terms of underlying mechanisms, they have some phenomenological aspects and potential applications in common. Electron transfer phenomena occur between electronically separated fluorophores, in which their relative distance, orientation and mobility play instrumental roles. Therefore, some of these applications include molecular dynamics measurements, e.g., interaction between molecules or conformational changes within molecules, various reporter assays, bio-imaging, boosting or directing photoluminescence in both organic molecules and inorganic nanostructures such as zinc oxide quantum dots, solar collectors, and in semiconductors such as organic light-emitting diodes (OLEDs). ${ }^{1-5}$

FRET (Fig. 1a) involves non-radiative energy transfer from the excited state donor fluorophore to an acceptor molecule via Coulomb interaction. ${ }^{6,7}$ FRET relies on close donor and acceptor (D-A) proximity, scales with an inverse 6th power law,

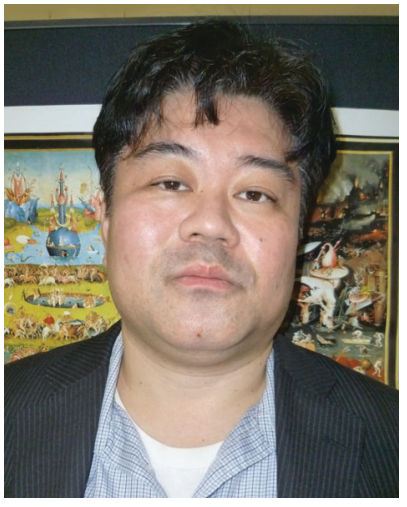

Gen-ichi Konishi
Gen-ichi Konishi was born in Rochester, NY, USA in 1971. He received his BSc degree from Osaka Prefecture University under the supervision of Professor Kazuhiko Mizuno in 1995 and his PhD from Kyoto University, Department of Polymer Chemistry, in 2000 under the supervision of Professor Yoshiki Chujo. Immediately thereafter he joined Shinshu University School of Medicine, where he worked as an assistant professor for 2 years in the Division of Physiology. He became an assistant professor in the Department of Chemistry \& Chemical Engineering, Kanazawa University in 2002 and was promoted to associate professor in the Department of Organic and Polymeric Materials, Tokyo Institute of Technology in 2006. He was appointed JST PRESTO researcher (Element Strategy; mentor: Professor Hideo Hosono). His research interests include photochemistry, fluorescent dyes, redox-photosensitised reactions (photoredox catalysis), polymer synthesis, optical materials, and physiology of the autonomic nervous system. requires spectral overlap of donor emission and acceptor absorption spectra, and depends on the relative orientation of the donor emission and acceptor absorption dipole moments. Next to energy transfer between species, various other processes can also deplete the donor's excited state, thereby changing the fluorophores' emission characteristics. Dexter energy transfer $(\mathrm{DET})^{8}$ is an exchange process in which two molecules (intermolecular) or two parts of the same molecule (intramolecular) bilaterally exchange their electrons (Fig. 1b). DET requires orbital overlap between donor and acceptor and scales with an $\mathrm{e}^{-R} \mathrm{D}-\mathrm{A}$ distance dependence. ${ }^{8}$ Charge transfer processes include the aforementioned excimer and exciplex formation, ${ }^{1,9,10}$ which are short-lived homodimers (excimer) or heterodimers (exciplex) of which at least one molecule is in the excited state. Such complexes occur via electrostatic attraction because of partial charge transfer between the individual entities and show red-shifted emission compared with the monomer's emission.

While the aforementioned processes all reflect relative arrangements of fluorophores, fluorescence phenomena governed by Twisted Intramolecular Charge Transfer (TICT) involve conformations of an individual fluorophore. TICT is a relatively common phenomenon in molecules that consist of a D-A (be advised that $\mathrm{D}$ and $\mathrm{A}$ in the context of charge transfer refers to electron donating or accepting groups rather than excitation energy donors or acceptors) linked by a single bond (Fig. 1c). ${ }^{12}$ In polar environments, such fluorophores undergo fast intramolecular electron transfer from the donor to the acceptor part of the molecule. This electron transfer is accompanied by intramolecular D-A twisting around the single bond (Fig. 1c) and produces a relaxed perpendicular structure. The equilibration between a relaxed perpendicular conformer and a coplanar conformer often results in dual fluorescence, i.e., from a high energy band through relaxation of the locally excited (LE) state and from a lower energy band due to emission from the TICT state. Since the relaxation pathways can easily be modulated by substituents, local polarity and steric restrictions, the TICT process can be exploited for novel design strategies of functional molecules. Therefore, TICT fluorescence holds great promise in applications such as OLEDs, chemosensors, and photovoltaic devices. This compact review examines some of the most recent advances in TICT research from the viewpoint of materials chemistry and potential applications in life science research, biomedical imaging and diagnostics, in optoelectronic devices, and photovoltaic applications.

\section{Fundamentals of TICT}

The seminal review by Grabowski, Rotkiewicz and Rettig compiled intuitive, but insightful information and images of TICT formation, and forms an essential basis for engineering various materials that directly exploit connected D-A systems. ${ }^{12}$ On an adiabatic energy surface of the charge transfer (CT) state of an excited D-A system, two disparate excited states exert opposite forces; the force that twists a D-A junction has an electron transfer (ET) character, whereas the force that prefers a coplanar conformation arises from mixing with a locally excited $\left({ }^{1}\right.$ LE) state (Fig. 2). 

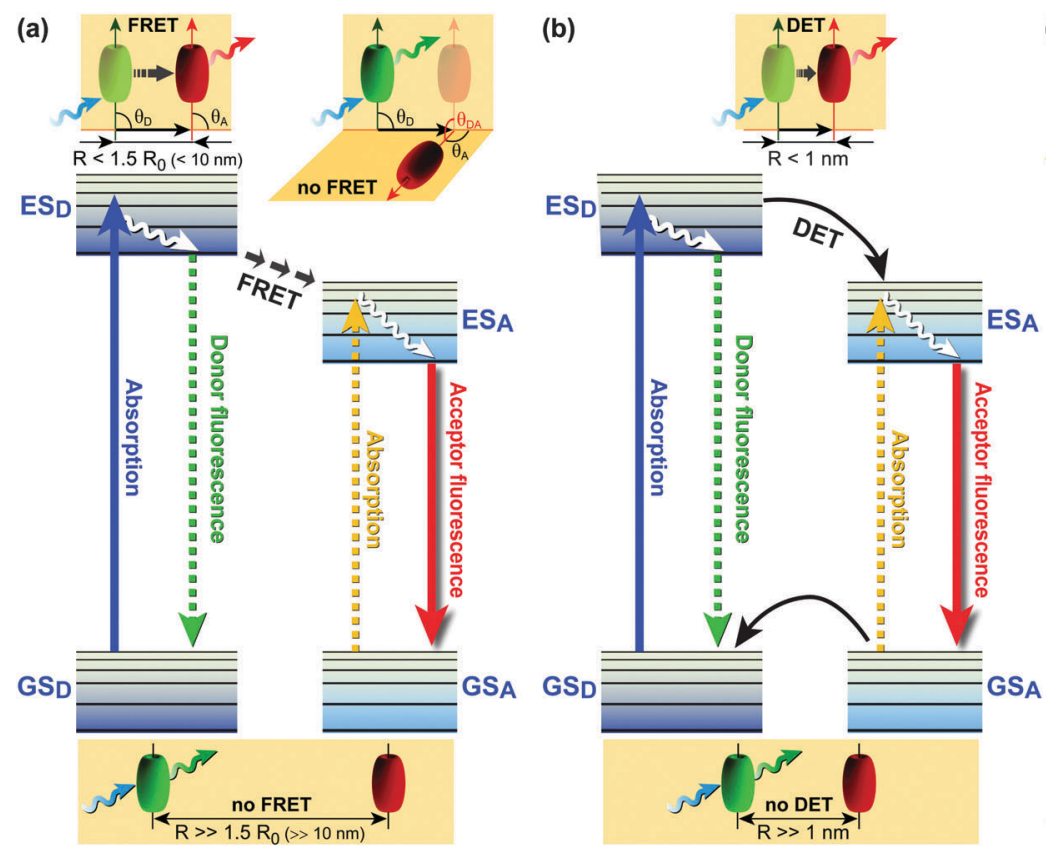

(c)

Fig. 1 Jabłoński diagrams of various energy/electron donor-acceptor ( $D-A)$ systems. (a) Förster Resonance Energy Transfer (FRET). $\theta$ : angle between vectors of the donor emission and the acceptor absorption; (b) Dexter Energy Transfer (DET); (c) Twisted Intramolecular Charge Transfer (TICT) dynamics. ${ }^{11}$ Upon excitation from the GS, the LE state equilibrates rapidly with the TICT state after fast charge transfer. GS = ground state; GS $=$ ground state donor; $\mathrm{GS}_{\mathrm{A}}=$ ground state acceptor; $\mathrm{ES}_{\mathrm{D}}=$ excited singlet state donor; $\mathrm{ES}_{\mathrm{A}}=$ excited singlet state acceptor; $\mathrm{LE}=$ locally excited state; $\mathrm{R}=$ effective D-A distance.

The ${ }^{1} \mathrm{ET}$ state is associated with a pair of frontier orbitals, i.e. the HOMO and LUMO where a single electron is transferred from the HOMO to the LUMO. As for D-A systems, an electron is transferred from the donor orbital (HOMO) to the acceptor orbital (LUMO) upon photoexcitation resulting in a biradicaloid pair (Fig. 2a). Frontier orbital interaction increases the excitation energy required to reach the ${ }^{1} \mathrm{ET}$ state and consequently a perpendicular conformation minimises the excitation energy. At a perpendicular conformation, the relative energy level of the ${ }^{1} \mathrm{ET}$ state can be approximated by subtracting the electron

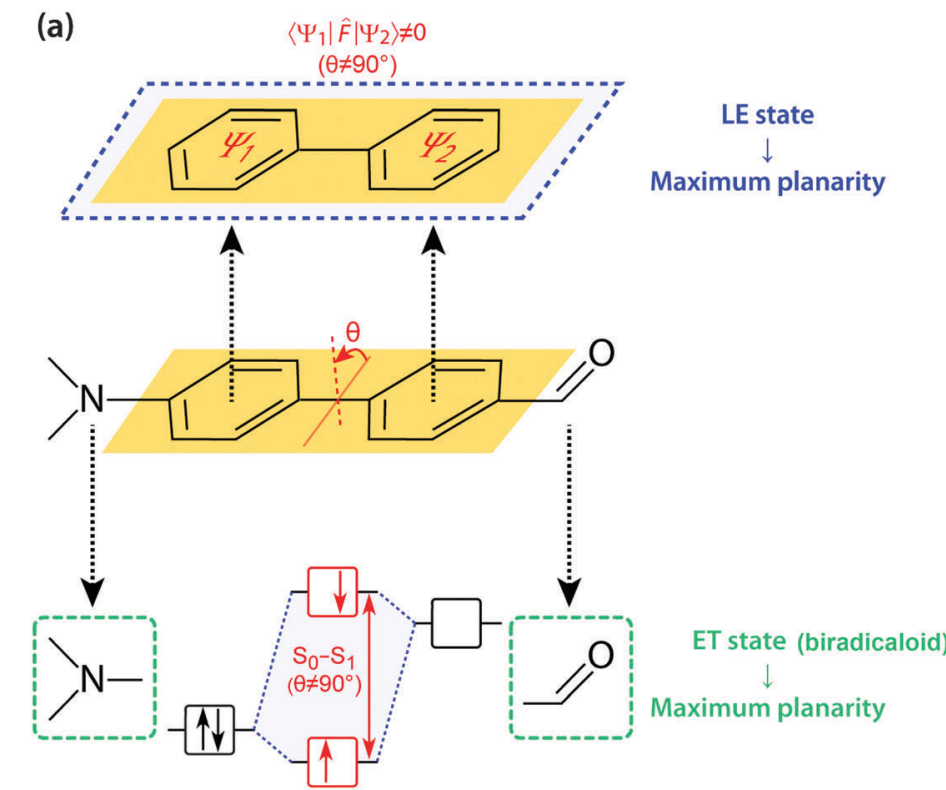

(b)

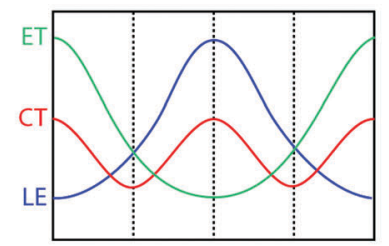

(c)
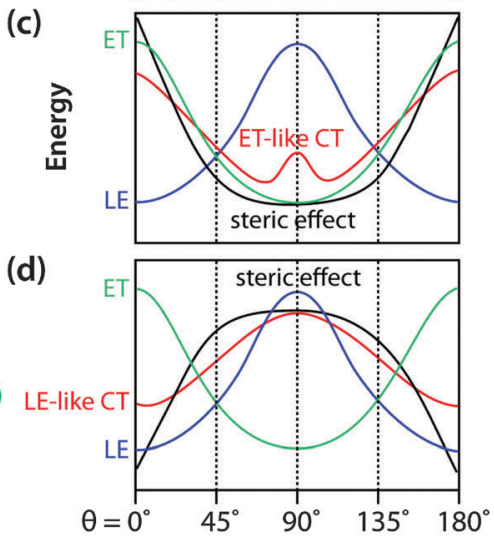

Fig. 2 (a) Preferred geometries of ${ }^{1} \mathrm{LE}$ and ${ }^{1} \mathrm{ET}$ states. Schematic energy diagrams of the ${ }^{1} \mathrm{LE}$ (blue), ${ }^{1} \mathrm{ET}$ (green) and ${ }^{1} \mathrm{CT}$ (red) states when: (b) ${ }^{1} \mathrm{LE}$ and ${ }^{1} \mathrm{ET}$ character are comparable to each other, (c) when the steric restriction (black) is introduced to twist the D-A junction (e.g. an alkyl group at orthoposition) and (d) when the D-A junction is made coplanar (e.g. a carbon bridge). 
affinity of an acceptor orbital from the ionisation potential of the donor orbital and thus strong donors and acceptors stabilise the ${ }^{1}$ ET state. The ${ }^{1}$ ET state is not only ubiquitously found in $\mathrm{D}-\mathrm{A}$ systems but also in those with isomerisable double bonds. In contrast, mesomeric interaction between $\pi$-subsystems $\left(\Psi_{1}\right.$ and $\Psi_{2}$ ) stabilises the ${ }^{1} \mathrm{LE}$ state in a coplanar conformation (Fig. 2a). Resonance stabilisation and accompanying planarisation between $\pi$-subsystems is particularly prompted in the excited state; multiple interactions between whole occupied and unoccupied $\pi$-orbitals of $\Psi_{1}$ and $\Psi_{2}$ enhance the HOMO level and reduce the LUMO level resulting in a lower ${ }^{1} \mathrm{LE}$ level. These competing two forces divide the ${ }^{1} \mathrm{CT}$ surface to generate either single or multiple minima (Fig. 2b). When the ET character of ${ }^{1} \mathrm{CT}$ outweighs the LE character of $\mathrm{S}_{1}$, the ${ }^{1} \mathrm{CT}$ minimum distinctly becomes a TICT state.

The relatively simple concept that governs TICT opens up a myriad of possibilities for designing novel functional molecules; the competition between LE and ET character of ${ }^{1} \mathrm{CT}$ can easily be manipulated by adjusting several factors, including steric restrictions, polarity environments, and D-A efficacy and strength. It is the sensitivity towards conformation and the surrounding environment that makes D-A systems attractive for various applications. For example, the introduction of steric hindrances such as an alkyl group at the ortho-position of the D-A junction deforms the ${ }^{1} \mathrm{CT}$ surface to locate its minima at a severely twisted conformation (Fig. 2c). In this conformation, the ${ }^{1} \mathrm{LE}$ state is too destabilised to mix efficiently with the ${ }^{1} \mathrm{ET}$ state and therefore, the ${ }^{1} \mathrm{CT}$ minimum becomes a highly twisted and polarised state (i.e. TICT state). On the other hand, when a steric restriction such as a carbon bridge between a donor and an acceptor forces the D-A junction to be coplanar, the ${ }^{1} \mathrm{LE}$ state is sufficiently stabilised so that the ${ }^{1} \mathrm{CT}$ minimum is governed by ${ }^{1} \mathrm{LE}$ character (i.e. coplanar ICT state, Fig. 2d). The polarity of the surrounding environment brings about similar effects with regard to ${ }^{1} \mathrm{ET}-{ }^{1} \mathrm{LE}$ mixing and such a polarity effect can be employed to design fluorescent agents for environmental sensing.

\section{Sensing environmental polarity}

Because a stronger ET character translates into a more pronounced charge separation, TICT conformations are distinctly induced in polar environments and by substitution of strong donors and acceptors. For example, Nagano and co-workers developed a library of boron-dipyrromethene (BODIPY)-based environmental polarity sensors (1) (Fig. 3), whose donors, when the polarity of an environment surpasses a particular ON/OFF threshold, quench the LE fluorescence of BODIPY via electron-transfer reactions. ${ }^{13}$ The BODIPY core is generally only moderately sensitive to environmental changes. ${ }^{14}$ Transitions from fluorescent, but less polarised states dominated by LE states, to non-fluorescent and highly polarised TICT states are also driven by the introduction of steric restrictions; the so called "pretwisting". Since the transition dipole moment of the ${ }^{1} \mathrm{LE}$ state of BODIPY and polarisation of the ${ }^{1} \mathrm{ET}$ state of (1) are perpendicular to each other, ${ }^{1} \mathrm{ET}-{ }^{1} \mathrm{LE}$ mixing does

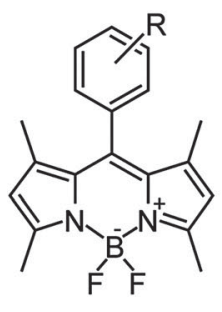

(1)<smiles>Cc1cc(C)c(B(c2c(C)cc(C)cc2C)n2c(-c3ccccc3)ccc2-c2ccccc2)c(C)c1</smiles>

(4)

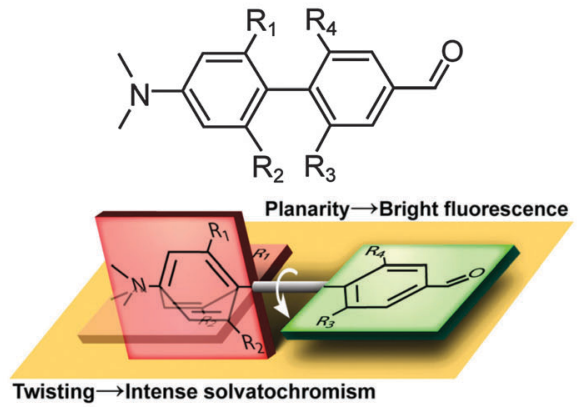

(2)<smiles>Cc1ccccc1N(c1ccccc1C)c1c2ccccc2c(N(c2ccccc2C)c2ccccc2Cl)c2ccccc12</smiles>

(5)<smiles>N#C/C(=C\c1cc2c3c(c1)CCCN3CCC2)C(=O)O</smiles>

(6)<smiles>Cc1ccc2c(c1)sc(-c1ccc(N(C)C)cc1)[n+]2C</smiles>

(7)<smiles>O=Cc1ccc(-c2ccc(N3CCCCC3)c3ccccc23)c2ccccc12</smiles>

(3)<smiles>[R]c1cccc(C=C(C#N)c2cccc([R])c2)c1</smiles>

(8)

Fig. 3 Examples of fluorescent molecules that utilise twisted intramolecular charge transfer (TICT) phenomena, as discussed in this review. (1) 4,4-Difluoro-8-(Rphenyl)-1,3,5,7-tetramethyl-4-bora-3a,4a-diaza-s-indacene; (2) $R_{1}, R_{2}, R_{3}, R_{4}$-substituted 4-formyl-4'-N,N-dimethylamino-1,10-biphenyl; schematic representation of solvatochromism in push-pull biphenyls; (3) 4-formyl-4'-piperidyl-1,1'-binaphthyl (a dual-mode fluorescent $D-\pi$-A-type biaryl dye); (4) 1-[bis(2,4,6trimethylphenyl)boranyl]-2,5-diphenyl-1H-pyrrole; (5) diarylaminoanthra-cenes; (6) 9-(2-carboxy-2-cyano vinyl)julolidine; (7) thioflavin T (2-[4-(dimethylamino)phenyl]-3,6-dimethyl-1,3-benzothiazol-3-ium chloride); (8) $\alpha$-cyanostilbene derivatives. 
not affect ${ }^{1} \mathrm{LE}$ fluorescence of BODIPY just before the ON/OFF threshold. Exploiting this principle, excited-state dipole moments and the resultant solvatochromisms - the ability to change color in response to changes in solvent polarity - of push-pull biphenyls (2) were modulated simply by imposing torsional restrictions. ${ }^{15}$ When a LE state cannot be mixed with a ${ }^{1} \mathrm{ET}$ (or ${ }^{1} \mathrm{CT}$ ) state for symmetry reasons, the ${ }^{1} \mathrm{LE}-{ }^{1} \mathrm{CT}$ interconversion results in fluorescence properties that are highly sensitive to steric environments, i.e., aggregation and rigidification of media, which we recently exemplified in the push-pull binaphthyl (3) shown in Fig. $3{ }^{16}$ However, it is worth mentioning that TICT formations do not always impose weak fluorescence. As comprehensively reviewed by Grabowski et al., ${ }^{12,17}$ if the structural relaxation of excited states involves more than one bond simultaneously, the resulting TICT states may acquire sizable fluorescence quantum yields. ${ }^{18}$ Recently, Yamaguchi's group reported a striking example of this phenomenon in $N$-borylated 2,5-diarylpyrroles (4). ${ }^{19}$ Similarly, we applied this concept by extending the $\pi$-conjugation of the widely used dopant for organic light emitting diodes (OLED), 9,10bis( $N, N$-diarylamino)anthracene (5); distortion of the donor $\left(N, N\right.$-diarylamine) plane destabilises the ${ }^{1} \mathrm{ET}$ state and the resultant mixing with the LE state enhances the oscillator strength of $\mathrm{S}_{0} \leftarrow{ }^{1} \mathrm{CT}$ fluorescence. ${ }^{20}$

As mentioned above, torsional restrictions in $\mathrm{D}-\mathrm{A}$ systems enable precise control over the proportion of the LE and ET character of the D-A system. Therefore, the pretwisting strategy must be effective when designing solvatochromic probes ${ }^{15,21}$ and photoinduced electron transfer (PET) sensors, ${ }^{22}$ in spite of the limited number of reports.

\section{Sensing microenvironmental viscosity}

Depending on the proportion of ET and LE character, directly connected D-A systems drastically change their fluorescence emission wavelengths, quantum yields, lifetimes, and spectral shapes. These dynamic characteristics are highly useful for fluorescence imaging of microenvironmental viscosity, especially in biological systems. The particular class of TICT-active fluorophores called "molecular rotors" can enhance their fluorescence intensity in sterically restricted environments such as viscous media. ${ }^{23}$ Viscous environments make TICT-active fluorophores unable to overcome potential barriers against ${ }^{1}$ LE-TICT interconversions or, in some cases, viscosity hampers internal conversion from the TICT state. Representative molecular rotors (Fig. 3) are 9-(2-carboxy-2-cyanovinyl)julolidine (6) and its analogues, ${ }^{24,25}$ whose versatilities have been demonstrated not only in molecular biology, such as in peptide-protein interaction studies, ${ }^{26}$ imaging intracellular microviscosity, ${ }^{27}$ and amyloid deposit research, ${ }^{28}$ but also in polymer science, ${ }^{29}$ contact mechanics, ${ }^{30}$ and fluid dynamics. ${ }^{31}$ Thioflavin-T (7) is a well-known TICT-based molecular rotor used in in vitro cell biological and biomedical assays, in particular in drug discovery assays and mechanistical studies with respect to amyloid-related neurodegenerative diseases. ${ }^{32}$ Though there are other emerging molecular rotors, such as $\alpha$-cyanostilbene derivatives $(8)^{33}$ and various PET probes, ${ }^{34}$ we cannot cover the numerous articles on molecular rotors here, and therefore, we refer the reader to more specialised reviews on molecular rotors. ${ }^{23,24,31,35,36}$

\section{Sensing chemical species}

The presence of specific chemical species can also be visualised with TICT-active fluorophores such as RhoNox-1. ${ }^{37}$ RhoNox-1 (9) is the $N$-oxide derivative of Rhodamine B (Fig. 4a) and normally exhibits weak fluorescence due to TICT formation, as exemplified in the micrograph of Fig. 4b. However, in the presence of $\mathrm{Fe}^{2+}$ ions, this $\mathrm{N}$-oxide moiety in RhoNox-1 (9) is selectively reduced to yield highly fluorescent Rhodamine B (Fig. 4c). Hirayama et al. determined that the observed fluorescence intensity enhancement directly correlated with the concentration of $\mathrm{Fe}^{2+}$ ions, whilst treatment with various reactive oxygen species did not significantly enhance RhoNox-1's fluorescence.

In another approach, Xie and co-workers developed "turnon" $\mathrm{CN}^{-}$probes (10a-c; Fig. 5a) by introducing dicyanovinyl units at sterically demanding positions of large $\pi$ frameworks, thereby forcing the moieties to twist out of the anthryl plane. ${ }^{38}$ On such a highly pretwisted D-A system, the ET character becomes dominant and the system's fluorescence is severely quenched through TICT formation (Fig. 2c). Nucleophilic addition of $\mathrm{CN}^{-}$ to dicyanovinyl groups disables their electron-accepting abilities, and fluorescence is thus drastically and concentration-dependently enhanced (Fig. 5b). In these "turn-on" type fluorescent probes, the strength of either donors or acceptors is determined through chemical reaction with the analyte. Similar strategies, by combining bulky diphenylamino and 9-anthryl units or in a system through $\mathrm{H}$-bonding and charging of aromatic building blocks, have also been adopted in the development of hydrazine $\left(\mathrm{H}_{2} \mathrm{NNH}_{2}\right)$ sensing probes. ${ }^{39,40}$

Nakamura and co-workers adopted a significantly different approach to detect specific chemical species. They discovered that anthracene anilide derivatives undergo fluorescence quenching via TICT formation, which can partially be suppressed in the presence of alkaline-earth-metal ions. ${ }^{41}$ To amplify the sensitivity of anthracene anilides, two anthracene anilides were connected with a linear polyether ((11); Fig. 5c) in order to fixate its conformation and suppress torsional motion around the $\mathrm{Ph}-\mathrm{NH}-\mathrm{CO}-$ bond axis upon complexation with metal ions. As a consequence, (11) exhibited a 50-70-fold increase in its fluorescence intensity by the addition of $\mathrm{Ca}^{2+}, \mathrm{Sr}^{2+}$ or $\mathrm{Ba}^{2+}$ ions. ${ }^{42}$ This strategy, wherein complexation prevents TICT formation by imposing steric restriction, is also effective for the detection of neutral molecules such as surfactants. ${ }^{43}$

\section{Aggregation-induced emission luminogens}

Aggregation-induced emission (AIE) luminogens (AIEgens) are a class of fluorophores that display subtle fluorescence when dissolved in good solvents as molecules, but become highly fluorescent when clustered in poor solvents or in solid state as 


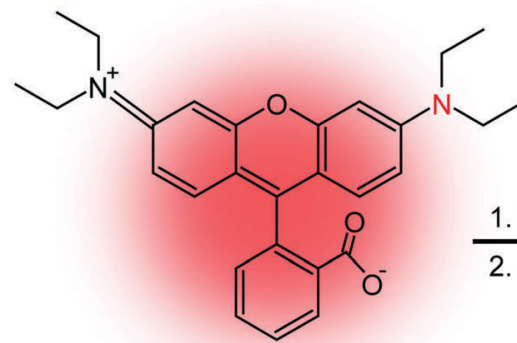

Rhodamine B strong fluorescence

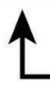

(b)

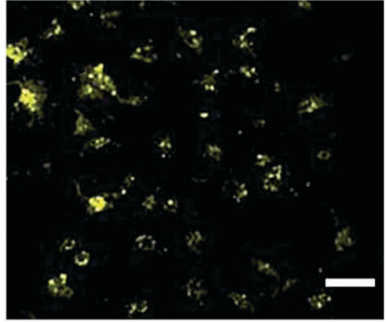

(a)<smiles>CCN(CC)C(=O)C1CCCCC1</smiles>

RhoNox-1

weak fluorescence

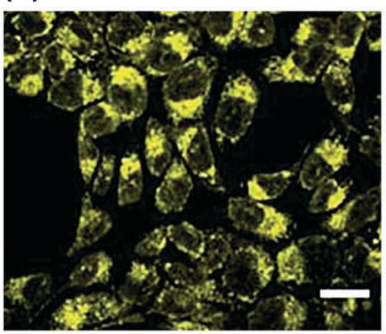

(d)

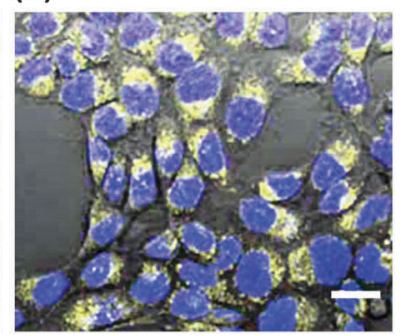

Fig. 4 (a) Structure of RhoNox-1 (9), synthesis from Rhodamine B by neutralisation ( $\mathrm{NaOH}$ ) and oxygenation with $m$-chloroperbenzoic acid ( $m$ - $\mathrm{CPBA}$ ), and mechanism of iron(॥)-ion detection. Bottom: Confocal fluorescence microscopy images of Fe(॥) detection with $\left.5 \mu \mathrm{M} \mathrm{RhoNox-1} \mathrm{(1} \mathrm{h,} 37^{\circ} \mathrm{C}\right)$ in HepG2 cells. (b) Control, (c) preincubated with $100 \mu \mathrm{M}$ Fe(॥) for $30 \mathrm{~min}$, and (d) bright field image merged with (c) and nuclear staining (Hoechst 3334) fluorescence micrographs. Bar: $20 \mu \mathrm{m}$. Adapted from ref. 37 with permission from the Royal Society of Chemistry (C) 2013.

aggregates. Since AIEgens were expected to be promising materials for optoelectronic and bioimaging applications, there has been a vast amount of relevant research, as compiled and comprehensively discussed in recent reviews. ${ }^{44-46}$ Therefore, in the current review, we will only discuss AIE from the viewpoint of TICT research.

Typically, AIE phenomena are predominantly caused by restriction of intramolecular motions (RIM) accompanied by aggregation. Intramolecular motions are also decisive in the photophysics of TICT-active fluorophores; the proportion of LE and ET character at $S_{1}$ (global or local) minima, potential barriers against LE-TICT interconversions, and non-radiative transition rates of TICT states are all governed by steric environments around fluorophores. ${ }^{12}$ Consequently, TICT-active fluorophores are ideal candidates for the development of unique AIEgens with exceptional properties (Fig. 6). In fact, tetraphenylethene (TPE; (12)), one of the most famous AIEgen ${ }^{47}$ is known to undergo TICT formation in an excited state. ${ }^{12,48,49} \mathrm{As}$ mentioned in the previous section, stilbene analogues, such as TPE, often possess strong ET character on their excited state. ${ }^{12}$ Taking ethylene as the simplest example, its $\mathrm{S}_{1}$ is a weakly polarized $\pi-\pi^{*}$ state in a coplanar conformation, whereas in a perpendicular conformation, $\mathrm{S}_{1}$ becomes a $\mathrm{p} \rightarrow \mathrm{p}$ orbital electron-transfer $\left({ }^{1} \mathrm{ET}\right)$ state, whose excitation energy is equal only to an electronic repulsion experienced by occupying the same orbital. Hence, the excited state of ethylene prefers to be a twisted ${ }^{50}$ and strongly polarized ${ }^{51}{ }^{1}$ ET state. Similarly, when mixing with the ${ }^{1}$ LE state (or mesomeric interaction between $\pi$-subsystems) is ignored and only HOMO-LUMO interaction determines the structure of $S_{1}$, molecules based on isomerisable double bonds, in principle, should undergo the TICT process. Since mixing from the ${ }^{1} \mathrm{LE}$ state should be weak on the propeller-shaped TPE (12), it is reasonable for TPE to be TICT active. Interestingly, when two phenyl rings of TPE are bridged by ether bonds retaining the $\mathrm{C}=\mathrm{C}$ bond can freely rotate, the bridged TPE recovers strong fluorescence even in solution state, which implies that the coplanar structure enhances mixing with the ${ }^{1} \mathrm{LE}$ state and thus hampered TICT formation occurs. ${ }^{52}$ So far, many AIEgens have been designed based on stilbene structures, as exemplified by $\alpha$-cyanostilbene derivatives (8), ${ }^{33,53-55}$ triphenylethene carbazole derivatives (13), ${ }^{56}$ and distyrylanthracene derivatives (14). ${ }^{57}$ Recently, we developed a novel AIE luminogen (15) based on a simple bis(piperidyl)anthracene structure, but which features unprecedented brightness and is highly pretwisted around $\mathrm{D}-\pi$ junctions. ${ }^{58}$

TICT-active D-A systems also sometimes exhibit AIE and, as mentioned previously, D-A systems based on BODIPY (1) are non-fluorescent in polar environments due to TICT formation, but recover their fluorescence intensity upon aggregation. ${ }^{59}$ Such a combination of polarity-reduced emissions and AIE was also discovered in push-pull binaphthyl (3) and barbituric acidfunctionalised tetraphenylethene derivatives ((16); TPE-HPhBar; Fig. 7), and revealed to be useful as optical waveguides. ${ }^{60}$ TPE-HPh-Bar shows a bright yellow emission at $545 \mathrm{~nm}$ upon UV excitation (Fig. 7a), which is quenched above a water (poor solvent) fraction of $60 \%$. Due to the increased solvent polarity, an intensity-reduced (see also Fig. 7b) and red-shifted emission 
(a)

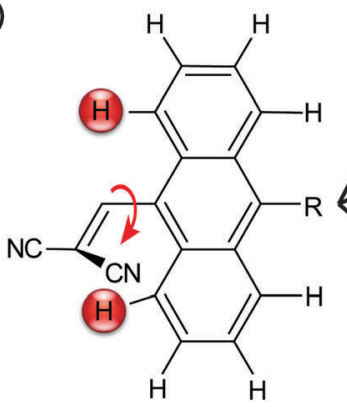

(10a-c)

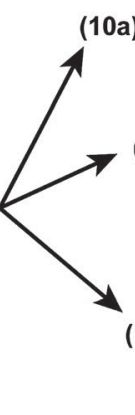

(10a): $\mathrm{H}$

(10b):<smiles>COc1ccc(C(C)(C)C)cc1</smiles>

(b)
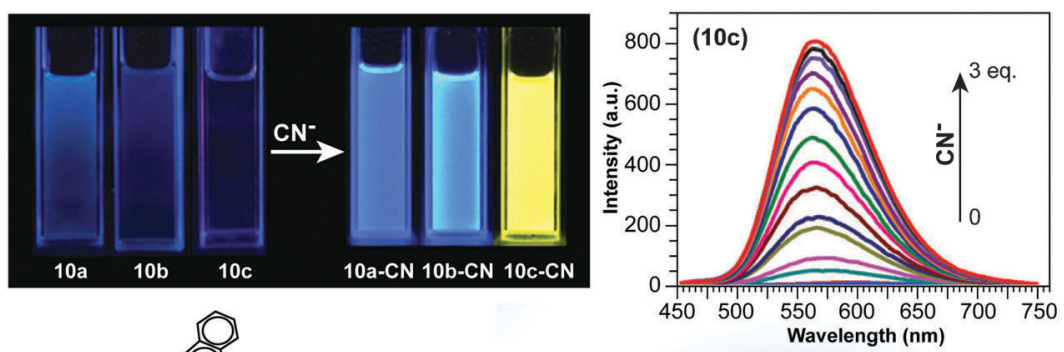

(c)
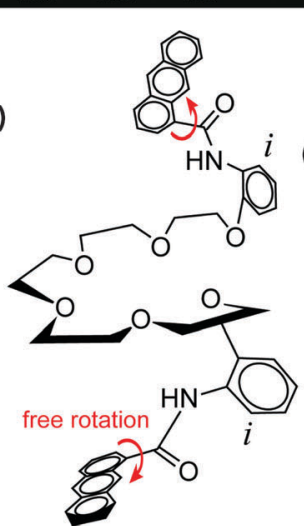

(11)<smiles>CC(C)(C)c1ccc(Nc2ccc(C(C)(C)C)cc2)cc1</smiles>
-n

Fluorescence: OFF

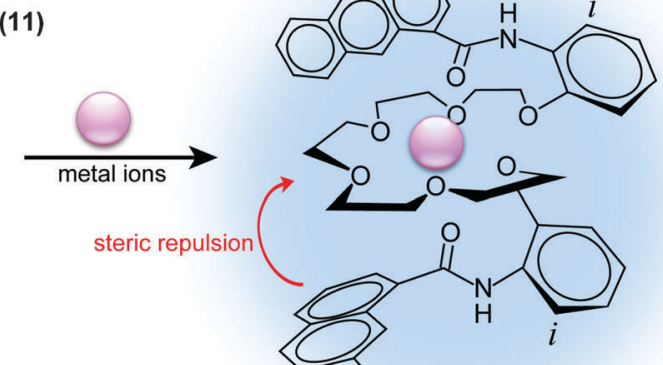

Fluorescence: ON

Fig. 5 (a) Chemical structures of "turn-on" $\mathrm{CN}^{-}$probes (9-dicyanovinyl anthracene) with various functional groups (10a-10c). (b) Fluorescence emission of $40 \mu \mathrm{M}$ of compounds $10 \mathrm{a}-\mathrm{c}$ under UV excitation with a portable lamp in the absence and presence of 3 eq. $\mathrm{CN}^{-}$. Right: Fluorescence changes upon addition of $\mathrm{CN}^{-}$to $10 \mathrm{c}\left(20 \mu \mathrm{M}, \lambda_{\mathrm{ex}}=396 \mathrm{~nm}\right)$ in $\mathrm{CH}_{2} \mathrm{Cl}_{2}$. (c) Proposed structural change of $\mathrm{N}, \mathrm{N}$-(ethylenedioxybis(3oxapentamethyleneoxy-2-phenyl))-bis(1-anthracenecarboxamide) in response to metal ions in the ground state. Adapted from ref. 38,42 with permission from the Royal Society of Chemistry (C)2013 and the Japan Society for Analytical Chemistry (C2009.

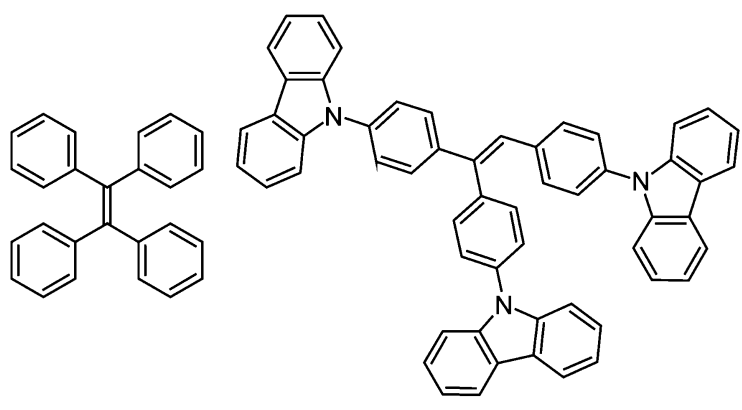

(12)<smiles>C(=C/c1c2ccccc2c(/C=C/c2ccccc2)c2ccccc12)\c1ccccc1</smiles>

(14)<smiles>c1ccc2c(N3CCCCC3)c3ccccc3c(N3CCCCC3)c2c1</smiles>

(15)

Fig. 6 Chemical structures of aggregation-induced emission (AIE) active dyes that utilise twisted intramolecular charge transfer (TICT): (12) tetraphenylethene; (13) 9-(4-(1,2-bis[4-(9H-carbazol-9-yl)phenyl]ethenyl)phenyl)-9H-carbazole; (14) 9,10-distyrylanthracene (DSA); (15) 9,10-bis(piperidyl)anthracene (9,10-BPA). 


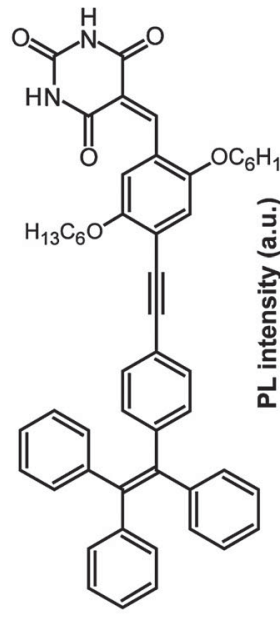

(16)
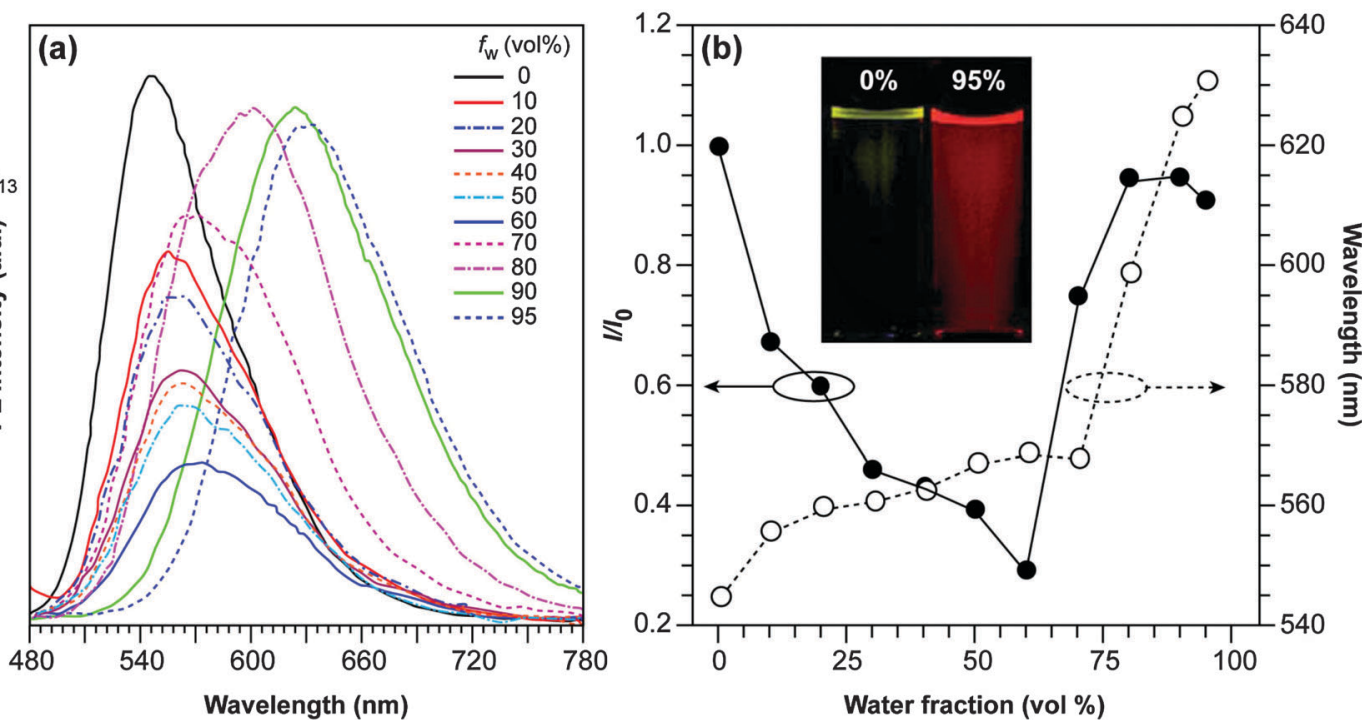

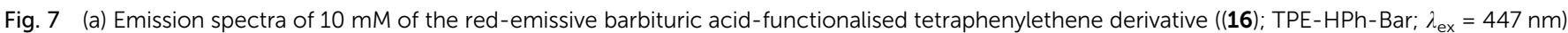

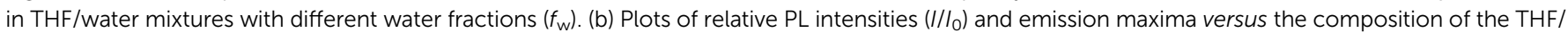

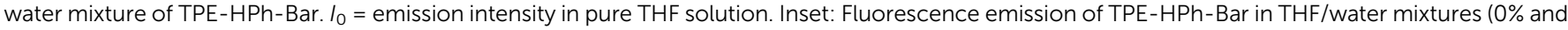
95\%) under UV excitation with a portable lamp. Adapted from ref. 60 with permission from the Royal Society of Chemistry C 2014.

is observed. Above $60 \%$, significant aggregation occurs and the AIE effect dominates the TICT effect with the consequence that the emission rises again (Fig. 7b). It is also noteworthy to mention that several AIEgens based on strong D-A systems, which are presumably TICT-active, have been reported to change their fluorescence properties upon external stimuli such as the exertion of external forces. ${ }^{61,62}$ These results imply that TICT-active molecules may potentially find applications in the field of fluorescence mechanochromism.

\section{Organic light emitting diodes}

The competition between LE character and ET character plays an instrumental role in tuning the electronic structure of dopants. In organic light-emitting diodes (OLED), the recombination of injected holes and electrons produces a so-called charge-transfer (CT) exciton, which decays to generate one photon directly, or relaxes to a low-lying and highly emissive locally excited (LE) exciton. To fully utilise both LE and CT excitons, Ma and co-workers designed the moderately twisted D-A system TPA-PPI ((17); Fig. 8); moderate torsion of TPA-PPI optimises the proportion of ${ }^{1} \mathrm{ET}$ and ${ }^{1} \mathrm{LE}$ character at the ${ }^{1} \mathrm{CT}$ minimum and consequently enables the ${ }^{1} \mathrm{CT}$ state not only to accept CT excitons but also to possess a large oscillator strength of LE excitons. ${ }^{63}$

The strongly mixed state, where the energy levels of LE and CT excitons are closely arranged, is called "hybridised local and charge transfer" (HLCT) state; an important strategy to enhance the electroluminescence efficiencies of OLEDs (Fig. 9a). ${ }^{64,65}$

Electrons and holes possess degrees of freedom not only in orbitals, but also in their spin. Thus, to maximise the quantum efficiency of OLEDs, $25 \%$ of singlet excitons and $75 \%$ of triplet excitons, generated by arbitrary recombination of carriers, must be utilised as either fluorescence or phosphorescence.
Thermally activated delayed fluorescence (TADF) paves the way for solving the problem; ${ }^{66}$ highly pretwisted D-A systems minimise ${ }^{1} \mathrm{CT}-{ }^{3} \mathrm{CT}$ energy gaps due to the absence of electron-exchange interactions. Initially populated ${ }^{3} \mathrm{CT}$ excitons are thus rapidly converted to their singlet counterparts. Therefore, TADF is compatible with TICT-active fluorophores. For example, $p$-TPA-3TPE- $p$ PhCN (18), which achieved high external quantum efficiency over its theoretical value when employing the TADF strategy, exhibited weak fluorescence in solution due to TICT formation and was highly emissive in the solid state. ${ }^{67}$ Recently fluorophores displaying both TADF and AIE were intensively investigated. ${ }^{68,69}$ Utilising long-wavelength fluorescence of TICT states, colour tuning of TADF emitters were demonstrated on phenoxazinesubstituted triphenyl-1,3,5-triazines (19). ${ }^{70}$

Recently, Adachi et al. achieved fast and efficient blue TADF based on engineering of LE and CT states. ${ }^{4}$ Since ${ }^{1} \mathrm{CT}$ and ${ }^{3} \mathrm{CT}$ corresponding to blue emissions lie at relatively high energy levels, the lowest triplet state $\left(\mathrm{T}_{1}\right)$ tends to become a ${ }^{3} \mathrm{LE}$ state and hamper reverse intersystem crossing from ${ }^{3} \mathrm{CT}$. To solve the problem of blue TADF, they arranged pretwisted D-A junctions at appropriate positions (20) in order to destabilise the ${ }^{3} \mathrm{LE}$ state (Fig. 9b). Taking Fig. 2a as an example, there are many D-A junctions (e.g. $N$-aryl, aryl-aryl, and aryl- $C$ (formyl) bonds) but only the torsion at the aryl-aryl effectively destabilises ${ }^{1} \mathrm{LE}$ and ${ }^{3} \mathrm{LE}$ states. Thus, the ${ }^{3} \mathrm{LE}$ state of DMAC-DPS (20) was proximal to the ${ }^{3} \mathrm{CT}$ and ${ }^{1} \mathrm{CT}$ states and underwent fast TADF. As a result, the device with (20) offered external quantum efficiencies of up to $19.5 \%$.

\section{Nonlinear optics}

Recently, progress in nonlinear optics (NLO) revitalised TICT research. It may sound strange that TICT states, which are formed in excited-state adiabatic reactions, affect NLO properties. 


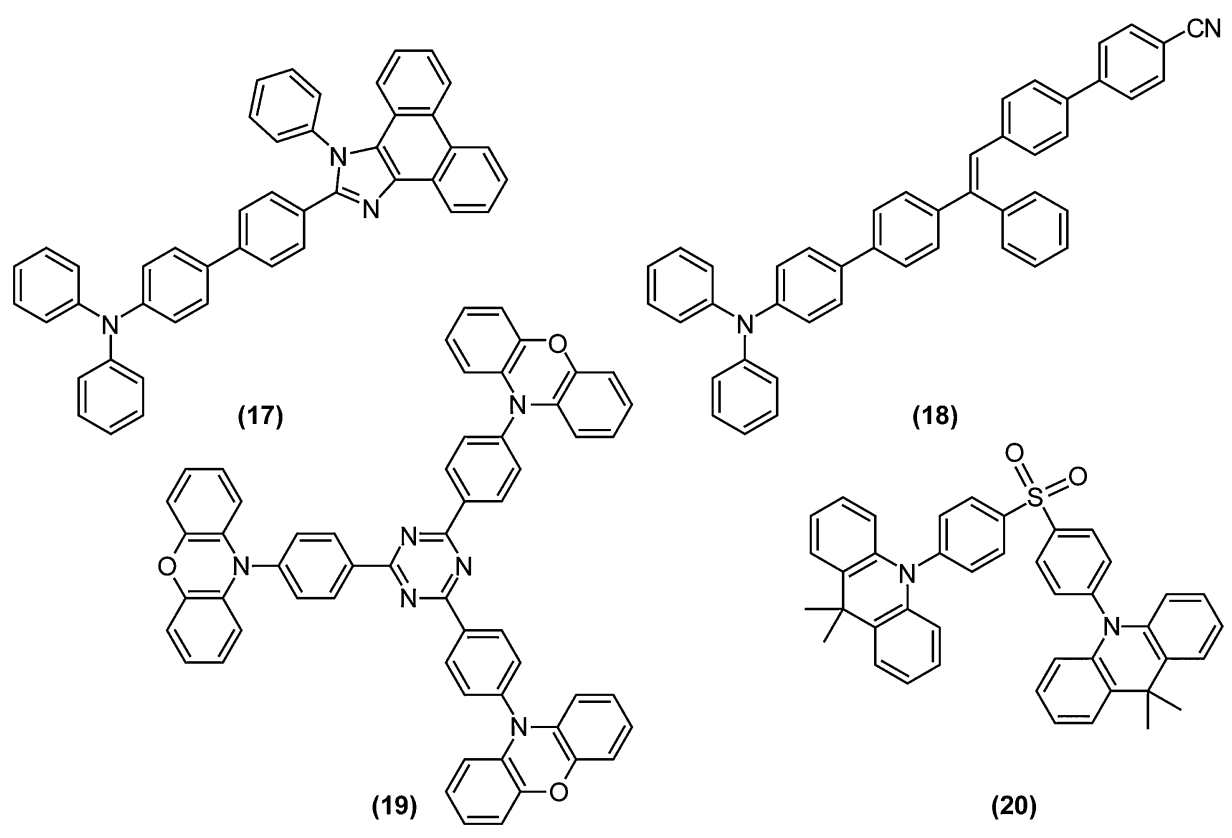

Fig. 8 Examples of compounds used in organic light emitting diodes (OLED) whose function is based on twisted intramolecular charge transfer (TICT): (17) N,N-diphenyl-4'-(1-phenyl-1H-phenanthro[9,10-d]imidazol-2-yl)biphenyl-4-amine (TPA-PPI); (18) 4-(4-[(E)-2-(4-[4-(diphenyl-amino)phenyl]phenyl)-2-phenylethenyl]phenyl)benzonitrile ( $p$-TPA-3TPE-p-PhCN); (19) 2,4,6-tri(4-(10H-phenoxazin-10H-yl)phenyl)-1,3,5-triazine (tri-PXZTRZ); (20) bis[4-(9,9-dimethyl-9,10-dihydroacridine)phenyl]sulfone (DMAC-DPS).

However, on highly pretwisted D-A systems, TICT-like states unquestionably exist with near-zero oscillator strengths and consequently affect NLO properties. In 1998, Ratner and co-workers theoretically predicted that highly twisted, but nonperpendicular, D-A systems would exhibit extraordinarily large second-order hyperpolarisabilities due to their minimal LE characters in $\mathrm{S}_{1}$ and large changes in dipole moments induced by photoexcitations. ${ }^{71}$ This theoretical research had a significant impact on design strategies for NLO materials, because at that time conventional dogma dictated that planar, rigid, and large $\pi$-systems were desirable. These nearly perpendicular $\mathrm{D}-\mathrm{A}$ systems, especially with zwitterionic structures, were named TICTOID chromophores. In 2005, Marks et al. first reported the synthesis of a TICTOID-type dye, i.e., "twisted $\pi$-electron system molecular chromophore" TMC-2 ((21); Fig. 10). ${ }^{72}$ TMC-2 displayed exceptional second-order hyperpolarisability $\beta$ and electrooptical (EO) response, far surpassing those of existing dyes $(\mu \beta=-488000 \times 10-48 \mathrm{esu}){ }^{72,73}$ Furthermore, TMC-2 (a)

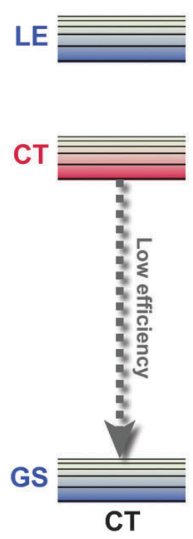

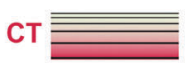

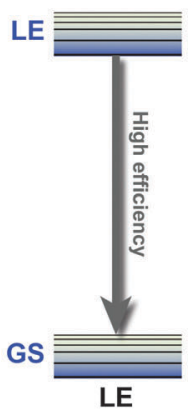

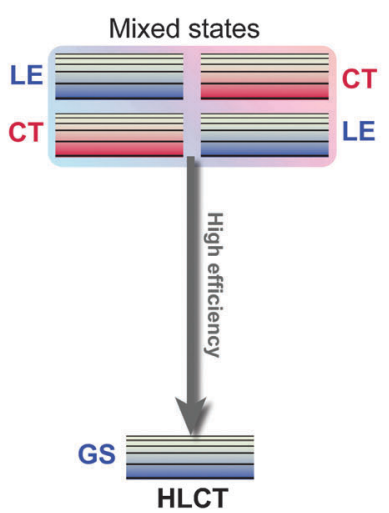

(b)

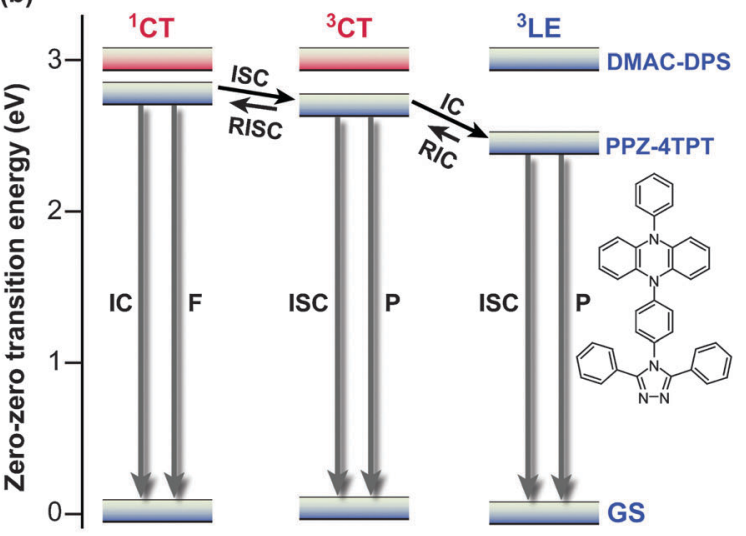

Fig. 9 (a) Conceptual illustration of the three possible local excited (LE) and charge transfer (CT) state energy levels in donor-acceptor (D-A) molecules, i.e., emission from CT, LE, and HLCT (hybridised local and charge transfer) states. (b) Energy levels of PPZ-4TPT (4-[4-(5-phenyl-5,10dihydrophenazine)phenyl]-3,5-diphenyl-1,2,4-triazole) and DMAC-DPS (bis[4-(9,9-dimethyl-9,10-dihydroacridine)phenyl]sulfone) calculated in toluene. F, fluorescence; P, phosphorescence; GS, ground state; IC, internal conversion; RIC, reverse internal conversion; ISC, intersystem crossing; RISC, reverse intersystem crossing. Adapted from ref. 4, 64 with permission from Macmillan Publishers Ltd: Nature Photonics and Wiley-VCH Verlag $\mathrm{GmbH} \&$ Co. KGaA (C)2014. 
<smiles>[R][n+]1cc(C)c(-c2c(C)cc(/C=C/c3ccc(C(C#N)C#N)cc3)cc2C)c(C)c1</smiles>

(21)

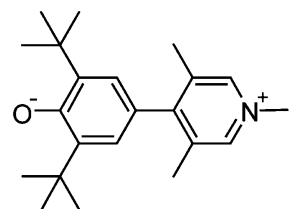

(23)<smiles>Cc1cc(C(C#N)C#N)cc(C)c1-c1c(C)c[n+](C2=C(/C=C/c3cc(-c4ccccc4)[s+]c(-c4ccccc4)c3)CCC/C2=C\C=C2C=C(c3ccccc3)SC(c3ccccc3)=C2)cc1C</smiles>

(22)

Fig. 10 Examples of non-linear optics (NLO) compounds that are based on twisted intramolecular charge transfer (TICT): (21) TMC, twisted $\pi$-electron system molecular chromophore: $R$-substituted 2-(4-[(E)-2-[4-(3,5-dimethylpyridin-4-yl)-3,5-dimethylphenyl]-ethenyl]phenyl)propanedinitrile; (22) Cy-TICT: contains 4-[4-(dicyanomethyl)-2,6-dimethyl-phenyl]-1-[(6E)-2-[(E)-2-(2,6-diphenyl-1 $\lambda^{4}$-thiopyran-1-ylium-4-yl)ethenyl]-6-[2-(2,6diphenyl-4H-thiopyran-4-ylidene)ethylidene]cyclohex-1-en-1-yl]-3,5-dimethyl-1 $\lambda^{5}$-pyridin-1-ylium; (23) 4-(3,5-di-tert-butyl-4-oxidophenyl)-1,3,5trimethylpyridin-1-ium.

was also shown to possess large third-order hyperpolarisability $\gamma$ only in real part, equivalent to the nonlinear refractive index. ${ }^{74}$ The effectiveness of TICTOID dyes is further demonstrated in various molecules, such as cyanine-TICTOID dyads $(\mathbf{2 2})^{75}$ and push-pull biphenyls (23). ${ }^{76}$

\section{Solar energy conversion with twisted D-A systems}

To collect and store solar energy in a stable form, a pair of charges generated by photoexcitation must be collected at electrodes or stored through formation of chemical bonds. Not surprisingly, the long lifetime of photoexcited states twisted D-A pairs such as (24) ${ }^{77}$ and $(\mathbf{2 5}),{ }^{78}$ as schematically depicted in Fig. 11.

Fukuzumi and co-workers realised efficient generation of a long-lived charge-separation (CS) state with a singly bonded D-A pair, i.e., 9-mesityl-10-methylacridinium ( Acr $^{+}-\mathrm{Mes}$; (26)). Severe steric hindrance in $\mathrm{Acr}^{+}$-Mes made the ET character advantageous, and thereby $\mathrm{Acr}^{+}-\mathrm{Mes}$ kept its perpendicular conformation even in the excited state. ${ }^{79}$ Furthermore, Acr $^{+}-$ Mes was shown to be active in several photocatalytic reactions. ${ }^{80,81}$ For instance, Acr ${ }^{+}-$Mes acts as an efficient photocatalyst in the selective bromination of aromatic hydrocarbons such as 1,2,4-trimethoxybenzene (TMB), with aqueous $\mathrm{HBr}$ as a $\mathrm{Br}$ source and $\mathrm{O}_{2}$ as an oxidant under visible light irradiation according to:

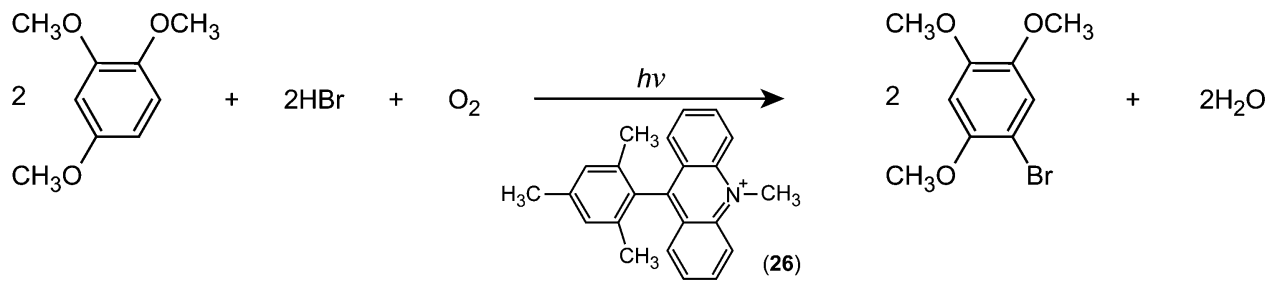

enabled by charge separation or triplet state generation plays an instrumental role in solar energy conversions. Typically, charge separations are studied in D-A systems in which the donor and acceptor are separated by an insulating bridge or spacer.

Parallel with research and developments in the TICT field, charge separation and transport dynamics were increasingly being studied; also in directly bonded D-A systems. Wasielewski and collaborators examined charge separation dynamics in several twisted D-A systems in which the donor and acceptor were directly bonded or connected by conjugating bridges. As a result, it was discovered that locally excited triplet states could efficiently be generated by charge recombination from highly
This photocatalytic bromination reaction was shown to proceed as schematically depicted in Fig. 12: (i) intramolecular photoinduced electron transfer from Mes to the singlet excited (SE) state of $\mathrm{Acr}^{+}\left(\mathrm{Acr}^{+}-\mathrm{Mes}\right)$ generates Acr--Mesc ${ }^{\bullet+}$. (ii) Electron transfer from TMB to $\mathrm{Mesc}^{\bullet+}\left(\mathrm{Acr}^{\bullet}-\mathrm{Mesc}^{\bullet+}\right)$ and from the Acr ${ }^{\bullet}$ moiety to $\mathrm{O}_{2}+\mathrm{H}^{+}$produces $\mathrm{HO}_{2} \bullet$. This reaction regenerates $\mathrm{Acr}^{+}-\mathrm{Mes}$ and the catalytic cycle for $\mathrm{Acr}^{+}-\mathrm{Mes}$ is at this point complete. (iii) The resulting TMB radical cation is able to react with $\mathrm{Br}^{-}$to form a $\mathrm{Br}$-adduct radical, which undergoes dehydrogenation with $\mathrm{HO}_{2}{ }^{\bullet}$ to yield brominated TMB and $\mathrm{H}_{2} \mathrm{O}_{2}$. (iv) However, $\mathrm{H}_{2} \mathrm{O}_{2}$ is able to react with $\mathrm{HBr}$ and TMB to yield yet another brominated TMB $+\mathrm{H}_{2} \mathrm{O}$ (Fig. 12). 


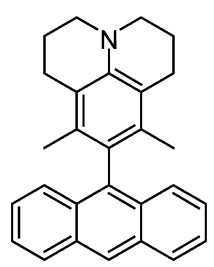

(24)

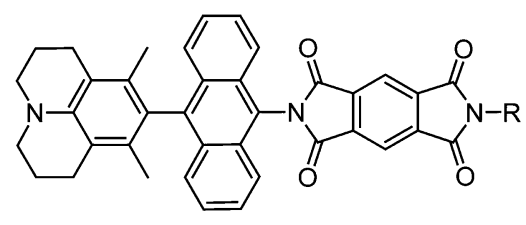

(25)

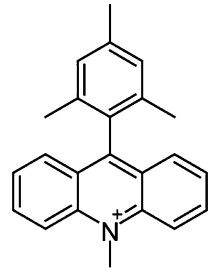

(26)

Fig. 11 Examples of functional dyes for solar energy conversion based on twisted intramolecular charge transfer (TICT): (24) 3,5-dimethyl-4-(9anthracenyl)julolidine; (25) 2-(10-(6,8-dimethyl-1-azatricyclo[7.3.1.0 5,13]trideca-5,7,9(13)-trien-7-yl)anthracen-9-yl)-6- $R$ - $1 H, 2 H, 3 H, 5 H, 6 H, 7 H-p y r r o l o[3,4-f]$ isoindole-1,3,5,7-tetrone; (26) 9-mesityl-10-methylacridinium (Acr $\left.{ }^{+}-\mathrm{Mes}\right)$.

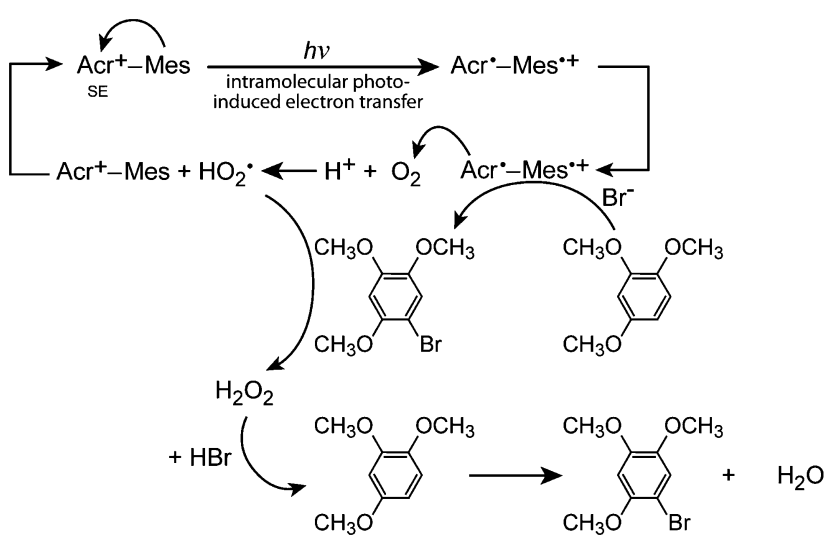

Fig. 12 Schematic representation of the photocatalytic bromination of 1,2,4-trimethoxybenzene (TMB) with 9-mesityl-10-methylacridinium $\left(\mathrm{Acr}^{+}-\mathrm{Mes}\right)$ as a catalyst. SE: Singlet excited state.

\section{Conclusions}

The foregoing examples have illustrated how researchers with various backgrounds have utilised the TICT phenomenon for purposes ranging from reporter assays and bioimaging to energy harvesting and display/lighting techniques. These examples also illustrate the strength of TICT, namely its tunability when donor, acceptor, and pendant groups are cleverly engineered. Such application-driven chemistry can only be performed well if the basic photophysical properties of TICT are sufficiently understood, as well as their relation to molecular structure. In this sense, TICT has matured significantly thanks to the large number of spectroscopic and theoretical studies that have been performed over the past decades. Nonetheless, from the perspective of materials chemistry, resolving a number of uncertainties would benefit the robustness of design strategies. For instance, in several molecular structures, the extent of pretwisting required to form the TICT state is unclear, data on excited-state CT equilibria are still incomplete due to the difficulty of discerning excited-state species from fluorescence spectra, the influence and contribution of other transfer effects such as exciplexes remains unclear, and various isomeric substitution effects have not yet been fully elucidated. Furthermore, a pile of theoretical insights accumulated so far have not yet been fully translated into design strategies for novel fluorophores. Therefore, it is important for TICT researchers both from material science and chemical physics to cooperate to fill these gaps for the benefit of developing novel fluorophores. Nonetheless, despite all this and the fact that numerous examples of TICT over a wide range of applications exist, it is comforting to know that only a relatively small number of design principles are involved. The future for TICT is bright!

\section{Conflicts of Interest}

G. P. C. D. is partially exempted from his duties by BNS to pursue fundamental scientific research. The authors declare no further conflicts of interest.

\section{Author contributions}

S. S drafted the review. G. P. C. D. and G.-i. K. revised and finalised the manuscript and G. P. C. D. made the figures. All authors approved the final manuscript.

\section{References}

1 H. C. Ishikawa-Ankerhold, R. Ankerhold and G. P. Drummen, Molecules, 2012, 17, 4047-4132.

2 J. Wu, W. Liu, J. Ge, H. Zhang and P. Wang, Chem. Soc. Rev., 2011, 40, 3483-3495.

3 Y. Zheng, C. Tan, G. P. Drummen and Q. Wang, Spectrochim. Acta, Part A, 2012, 96, 387-394.

4 Q. Zhang, B. Li, S. Huang, H. Nomura, H. Tanaka and C. Adachi, Nat. Photonics, 2014, 8, 326-332.

5 R. Zamiri and G. P. Drummen, in Concise Encyclopedia of Nanotechnology, ed. B. Kharissov, CRC Press, Boca Raton, 2016.

6 T. Förster, Naturwissenschaften, 1946, 6, 166-175.

7 T. Förster, Ann. Phys., 1948, 2, 55-75.

8 D. L. Dexter, J. Chem. Phys., 1953, 21, 836-850.

9 C. Lodeiro and F. Pina, Coord. Chem. Rev., 2009, 253, 1353-1383.

10 J. F. Callan, A. P. de Silva and D. C. Magri, Tetrahedron, 2005, 61, 8551-8588.

11 G. P. Drummen, Molecules, 2012, 17, 14067-14090.

12 Z. R. Grabowski, K. Rotkiewicz and W. Rettig, Chem. Rev., 2003, 103, 3899-4032.

13 H. Sunahara, Y. Urano, H. Kojima and T. Nagano, J. Am. Chem. Soc., 2007, 129, 5597-5604. 
14 G. P. Drummen, L. C. van Liebergen, J. A. Op den Kamp and J. A. Post, Free Radical Biol. Med., 2002, 33, 473-490.

15 S. Sasaki, Y. Niko, A. S. Klymchenko and G.-i. Konishi, Tetrahedron, 2014, 70, 7551-7559.

16 S. Sasaki, Y. Niko, K. Igawa and G.-i. Konishi, $R S C A d v$., 2014, 4, 33474-33477.

17 Z. R. Grabowski, Pure Appl. Chem., 1992, 64, 1249-1255.

18 R. Lapouyade, K. Czeschka, W. Majenz, W. Rettig, E. Gilabert and C. Rulliere, J. Phys. Chem., 1992, 96, 9643-9650.

19 T. Taniguchi, J. Wang, S. Irle and S. Yamaguchi, Dalton Trans., 2013, 42, 620-624.

20 S. Sasaki, K. Hattori, K. Igawa and G. Konishi, J. Phys. Chem. A, 2015, 119, 4898-4906.

21 S. Sumalekshmy and K. R. Gopidas, J. Phys. Chem. B, 2004, 108, 3705-3712.

22 B. Daly, J. Ling and A. P. de Silva, Chem. Soc. Rev., 2015, 44, 4203-4211.

23 M. A. Haidekker and E. A. Theodorakis, Org. Biomol. Chem., 2007, 5, 1669-1678.

24 M. A. Haidekker and E. A. Theodorakis, J. Biol. Eng., 2010, 4, 11.

25 J. Sutharsan, M. Dakanali, C. C. Capule, M. A. Haidekker, J. Yang and E. A. Theodorakis, ChemMedChem, 2010, 5, 56-60.

26 W. L. Goh, M. Y. Lee, T. L. Joseph, S. T. Quah, C. J. Brown, C. Verma, S. Brenner, F. J. Ghadessy and Y. N. Teo, J. Am. Chem. Soc., 2014, 136, 6159-6162.

27 K. Suhling, J. A. Levitt, P. H. Chung, M. K. Kuimova and G. Yahioglu, J. Visualized Exp., 2012, 2925, DOI: 10.3791/2925.

28 K. Cao, M. Farahi, M. Dakanali, W. M. Chang, C. J. Sigurdson, E. A. Theodorakis and J. Yang, J. Am. Chem. Soc., 2012, 134, 17338-17341.

29 R. D. Priestley, C. J. Ellison, L. J. Broadbelt and J. M. Torkelson, Science, 2005, 309, 456-459.

30 T. Suhina, B. Weber, C. E. Carpentier, K. Lorincz, P. Schall, D. Bonn and A. M. Brouwer, Angew. Chem., Int. Ed. Engl., 2015, 54, 3688-3691.

31 A. Mustafic, H. M. Huang, E. A. Theodorakis and M. A. Haidekker, J. Fluoresc., 2010, 20, 1087-1098.

32 N. Amdursky, Y. Erez and D. Huppert, Acc. Chem. Res., 2012, 45, 1548-1557.

33 L. Zhu and Y. Zhao, J. Mater. Chem. C, 2013, 1, 1059-1065.

34 T. Liu, X. Liu, D. R. Spring, X. Qian, J. Cui and Z. Xu, Sci. Rep., 2014, 4, 5418.

35 M. K. Kuimova, Chimia, 2012, 66, 159-165.

36 M. K. Kuimova, Phys. Chem. Chem. Phys., 2012, 14, 12671-12686.

37 T. Hirayama, K. Okuda and H. Nagasawa, Chem. Sci., 2013, 4, 1250-1256.

38 B. Chen, Y. Ding, X. Li, W. Zhu, J. P. Hill, K. Ariga and Y. Xie, Chem. Commun., 2013, 49, 10136-10138.

39 B. Chen, X. Sun, X. Li, H. Ågren and Y. Xie, Sens. Actuators, $B, 2014$, 199, 93-100.

40 D. Zhou, Y. Wang, J. Jia, W. Yu, B. Qu, X. Li and X. Sun, Chem. Commun., 2015, 51, 10656-10659.

41 T. Morozumi, T. Anada and H. Nakamura, J. Phys. Chem. B, 2001, 105, 2923-2931.
42 J. Kim, T. Morozumi, H. Hiraga and H. Nakamura, Anal. Sci., 2009, 25, 1319-1325.

43 Y. Oka, S. Nakamura, T. Morozumi and H. Nakamura, Talanta, 2010, 82, 1622-1626.

44 Y. Hong, J. W. Lam and B. Z. Tang, Chem. Soc. Rev., 2011, 40, 5361-5388.

45 J. Mei, Y. Hong, J. W. Y. Lam, A. Qin, Y. Tang and B. Z. Tang, Adv. Mater., 2014, 26, 5429-5479.

46 R. T. K. Kwok, C. W. T. Leung, J. W. Y. Lam and B. Z. Tang, Chem. Soc. Rev., 2015, 44, 4228-4238.

47 Y. Dong, J. W. Y. Lam, A. Qin, J. Liu, Z. Li, B. Z. Tang, J. Sun and H. S. Kwok, Appl. Phys. Lett., 2007, 91, 011111.

48 C. L. Schilling and E. F. Hilinski, J. Am. Chem. Soc., 1988, 110, 2296-2298.

49 T. Tahara and H.-O. Hamaguchi, Chem. Phys. Lett., 1994, 217, 369-374.

50 K. B. Wiberg, C. M. Hadad, J. B. Foresman and W. A. Chupka, J. Phys. Chem., 1992, 96, 10756-10768.

51 L. Salem, Acc. Chem. Res., 1979, 12, 87-92.

52 J. Shi, N. Chang, C. Li, J. Mei, C. Deng, X. Luo, Z. Liu, Z. Bo, Y. Q. Dong and B. Z. Tang, Chem. Commun., 2012, 48, 10675-10677.

53 Y. Gong, Y. Zhang, W. Z. Yuan, J. Z. Sun and Y. Zhang, J. Phys. Chem. C, 2014, 118, 10998-11005.

54 Y. Li, F. Li, H. Zhang, Z. Xie, W. Xie, H. Xu, B. Li, F. Shen, L. Ye, M. Hanif, D. Ma and Y. Ma, Chem. Commun., 2007, 231-233.

55 R. Wei, Y. He, X. Wang and P. Keller, Macromol. Rapid Commun., 2014, 35, 1571-1577.

56 Z. Yang, Z. Chi, T. Yu, X. Zhang, M. Chen, B. Xu, S. Liu, Y. Zhang and J. Xu, J. Mater. Chem., 2009, 19, 5541-5546.

57 J. He, B. Xu, F. Chen, H. Xia, K. Li, L. Ye and W. Tian, J. Phys. Chem. C, 2009, 113, 9892-9899.

58 S. Sasaki, K. Igawa and G.-i. Konishi, J. Mater. Chem. C, 2015, 3, 5940-5950.

59 R. Hu, E. Lager, A. Aguilar-Aguilar, J. Liu, J. W. Y. Lam, H. H. Y. Sung, I. D. Williams, Y. Zhong, K. S. Wong, E. PeñaCabrera and B. Z. Tang, J. Phys. Chem. C, 2009, 113, 15845-15853.

60 E. Wang, J. W. Y. Lam, R. Hu, C. Zhang, Y. S. Zhao and B. Z. Tang, J. Mater. Chem. C, 2014, 2, 1801-1807.

61 T. Han, Y. Hong, N. Xie, S. Chen, N. Zhao, E. Zhao, J. W. Y. Lam, H. H. Y. Sung, Y. Dong, B. Tong and B. Z. Tang, J. Mater. Chem. C, 2013, 1, 7314-7320.

62 N. Zhao, Z. Yang, J. W. Y. Lam, H. H. Y. Sung, N. Xie, S. Chen, H. Su, M. Gao, I. D. Williams, K. S. Wong and B. Z. Tang, Chem. Commun., 2012, 48, 8637-8639.

63 W. Li, D. Liu, F. Shen, D. Ma, Z. Wang, T. Feng, Y. Xu, B. Yang and Y. Ma, Adv. Funct. Mater., 2012, 22, 2797-2803.

64 W. Li, Y. Pan, L. Yao, H. Liu, S. Zhang, C. Wang, F. Shen, P. Lu, B. Yang and Y. Ma, Adv. Opt. Mater., 2014, 2, 892-901.

65 Z. Wang, Y. Feng, S. Zhang, Y. Gao, Z. Gao, Y. Chen, X. Zhang, P. Lu, B. Yang, P. Chen, Y. Ma and S. Liu, Phys. Chem. Chem. Phys., 2014, 16, 20772-20779.

66 H. Uoyama, K. Goushi, K. Shizu, H. Nomura and C. Adachi, Nature, 2012, 492, 234-238. 
67 J. Li, Y. Jiang, J. Cheng, Y. Zhang, H. Su, J. W. Y. Lam, H. H. Y. Sung, K. S. Wong, H. S. Kwok and B. Z. Tang, Phys. Chem. Chem. Phys., 2015, 17, 1134-1141.

68 S. Xu, T. Liu, Y. Mu, Y.-F. Wang, Z. Chi, C.-C. Lo, S. Liu, Y. Zhang, A. Lien and J. Xu, Angew. Chem., Int. Ed., 2015, 54, 874-878.

69 Z. Xie, C. Chen, S. Xu, J. Li, Y. Zhang, S. Liu, J. Xu and Z. Chi, Angew. Chem., Int. Ed., 2015, 54, 7181-7184.

70 H. Tanaka, K. Shizu, H. Nakanotani and C. Adachi, Chem. Mater., 2013, 25, 3766-3771.

71 I. D. L. Albert, T. J. Marks and M. A. Ratner, J. Am. Chem. Soc., 1998, 120, 11174-11181.

72 H. Kang, A. Facchetti, P. Zhu, H. Jiang, Y. Yang, E. Cariati, S. Righetto, R. Ugo, C. Zuccaccia, A. Macchioni, C. L. Stern, Z. Liu, S.-T. Ho and T. J. Marks, Angew. Chem., Int. Ed. Engl., 2005, 44, 7922-7925.

73 H. Kang, A. Facchetti, H. Jiang, E. Cariati, S. Righetto, R. Ugo, C. Zuccaccia, A. Macchioni, C. L. Stern, Z. Liu, S.-T. Ho, E. C. Brown, M. A. Ratner and T. J. Marks, J. Am. Chem. Soc., 2007, 129, 3267-3286.
74 G. S. He, J. Zhu, A. Baev, M. Samoć, D. L. Frattarelli, N. Watanabe, A. Facchetti, H. Ågren, T. J. Marks and P. N. Prasad, J. Am. Chem. Soc., 2011, 133, 6675-6680.

75 Y. Shi, A. J. T. Lou, G. S. He, A. Baev, M. T. Swihart, P. N. Prasad and T. J. Marks, J. Am. Chem. Soc., 2015, 137, 4622-4625.

76 A. Boeglin, A. Barsella, H. Chaumeil, E. Ay, J. Rotzler, M. Mayor and A. Fort, in Proc SPIE 7774, Linear and Nonlinear Optics of Organic Materials, 2010, pp. 777408-777410.

77 Z. E. X. Dance, S. M. Mickley, T. M. Wilson, A. B. Ricks, A. M. Scott, M. A. Ratner and M. R. Wasielewski, J. Phys. Chem. A, 2008, 112, 4194-4201.

78 M. T. Colvin, A. B. Ricks, A. M. Scott, D. T. Co and M. R. Wasielewski, J. Phys. Chem. A, 2012, 116, 1923-1930. 79 M. Hoshino, H. Uekusa, A. Tomita, S.-y. Koshihara, T. Sato, S. Nozawa, S.-i. Adachi, K. Ohkubo, H. Kotani and S. Fukuzumi, J. Am. Chem. Soc., 2012, 134, 4569-4572.

80 S. Fukuzumi, Phys. Chem. Chem. Phys., 2008, 10, 2283-2297. 81 K. Ohkubo, K. Mizushima, R. Iwata and S. Fukuzumi, Chem. Sci., 2011, 2, 715-722. 